\title{
Improving probabilistic image registration via reinforcement learning and uncertainty evaluation
}

\author{
Tayebeh Lotfi, Lisa Tang, Shawn Andrews and Ghassan Hamarneh \\ \{totfima, lisat, sda56, hamarneh\}@ sfu.ca \\ Medical Image Analysis Lab., Simon Fraser University, Burnaby, Canada
}

\begin{abstract}
One framework for probabilistic image registration involves assigning probability distributions over spatial transformations (e.g. distributions over displacement vectors at each voxel). In this paper, we propose an uncertainty measure for these distributions that examines the actual spatial displacements, thus departing from the classical Shannon entropy-based measures, which examine only the probabilities of these distributions. We show that by incorporating the proposed uncertainty measure, along with features extracted from the input images and intermediate displacement fields, we are able to more accurately predict the pointwise registration errors of an intermediate solution as estimated for a previously unseen input image pair. We utilize the predicted errors to identify regions in the image that are trustworthy and through which we refine the tentative registration solution. Results show that our proposed framework, which incorporates uncertainty estimation and registration error prediction, can improve accuracy of 3D image registrations by about $25 \%$.
\end{abstract}

\section{Introduction}

Image registration, the process of bringing images into spatial alignment, is a key step in the analysis of multi-modal image data. Formally, the process involves finding the optimal transformation $T$ that maps a point $\mathbf{v}_{i}$ in one image $I_{a}$ to corresponding point $\mathbf{v}_{i}+T\left(\mathbf{v}_{i}\right)$ in another image $I_{b}$. The task is often formulated as an energy minimization problem, e.g.:

$$
\underset{T}{\arg \min } \sum_{\mathbf{v}_{i} \in \Omega} \alpha D\left(I_{a}\left(\mathbf{v}_{i}\right), I_{b}\left(\mathbf{v}_{i}+T\left(\mathbf{v}_{i}\right)\right)\right)+R\left(T\left(\mathbf{v}_{i}\right)\right),
$$

where $\Omega \subset \mathbb{R}^{d}$ is the image domain, $d$ is the image dimension, $D$ measures the dissimilarity between image $I_{a}$ and the transformed image $I_{b}, R$ encourages the regularity of $T$, and $\alpha$ is a scalar balancing $D$ and $R$.

Formulating Eq. 1 as a graph labeling problem allows us to use efficient optimizers, such as graph cuts [13], and random walker (RW) [2]. In this work, we advocate the use of random walker optimization as it 1) provides a unique globally optimal solution that corresponds to an optimal spatial transformation, and 2) generates a probabilistic output, which we leverage to evaluate the solution's uncertainty.

In RW-based image registration (RWIR) [2], one samples the solution search space to generate a labelset $\mathcal{L}=\left\{\ell_{1}, \ell_{2}, \cdots, \ell_{L}\right\} \in \mathrm{R}^{d}$ (each label corresponds to a displacement vector). After optimization, one obtains a probabilistic, unity-sum vector 
$\mathcal{P}=\left\{p_{1}, \cdots, p_{L}\right\}$ at every $\mathbf{v}_{i}$. Given a probability distribution over the sampled (discrete and finite) space of possible displacements (or spatial transformations), one may calculate the registration uncertainty at $\mathbf{v}_{i}$ using Shannon's entropy:

$$
U_{\text {Shannon }}\left(\mathbf{v}_{i}\right)=-\sum_{i} p_{i}\left(\mathbf{v}_{i}\right) \log _{2}\left(p_{i}\left(\mathbf{v}_{i}\right)\right) .
$$

Although it is a natural choice, $U_{\text {Shannon }}$ however does not take into account the spatial meaning of displacement labels. For example, if two labels (i.e. two displacement vectors) are both assigned probability 0.5 , one would expect the uncertainty to depend on whether these labels were spatially close or far apart, but $U_{\text {Shannon }}$ ignores this. Therefore, it is critical to consider the spatial spread of the probability distribution over the displacement vectors. As our first contribution in this work, we develop an uncertainty measure tailored specifically for image registration that, in contrast to Eq. 2, takes into account the aforementioned spatial meaning of labels, as we will present in the next section.

Another important challenge in image registration is the evaluation of registration algorithms. Aside from qualitative visual inspection, the direct quantitative approach is to compare the deformation field with the ground truth (GT) transformation (at preselected landmark locations), yielding the target registration error (TRE). However, in clinical data, the GT is typically unknown and is hard to collect. To deal with the absence of GT, some methods opted to estimate registration accuracy, e.g., by using uncertainty measures as a surrogate for quantitative registration error. For instance, some methods [8,9] collected training data with GT warps (and hence registration error is known) and employed machine learning algorithms to infer registration error for novel data, while others $[7,12,11]$ used the inferred registration errors to improve the registration results. Adopting these research directions, our second contribution involves comparing how different uncertainty measures correlate with registration errors as we present in [10]. As our third contribution, we propose augmenting image- and deformation-based features with our novel uncertainty measure to add a new source of information that improves registration error prediction, and, for the first time, incorporate them into a probabilistic registration framework to boost its performance.

To this end, we developed a novel self-reinforcing registration framework that consists of the following steps: examine the local confidence of $T$ using our novel uncertainty measure; 2) employ random forest training to learn a predictive function $f$ that infers registration errors from a set of features that includes the proposed uncertainty measure; 3) given a tentative solution, employ $f$ to locate regions of high confidence (shown to correlate with low error), and utilize the displacements at those regions to refine the tentative solution in subsequent iterations.

\section{Method}

Our approach involves a training and testing stage. In the training stage, we learn a regression function $f$ that predicts registration error from a set of features (details later). In the testing stage, we use $f$ to predict the unknown registration error of a probabilistic registration and use locations with low predicted registration errors to steer subsequent registrations. Alg. 1 and Alg. 2 summarize the steps involved in our method. 


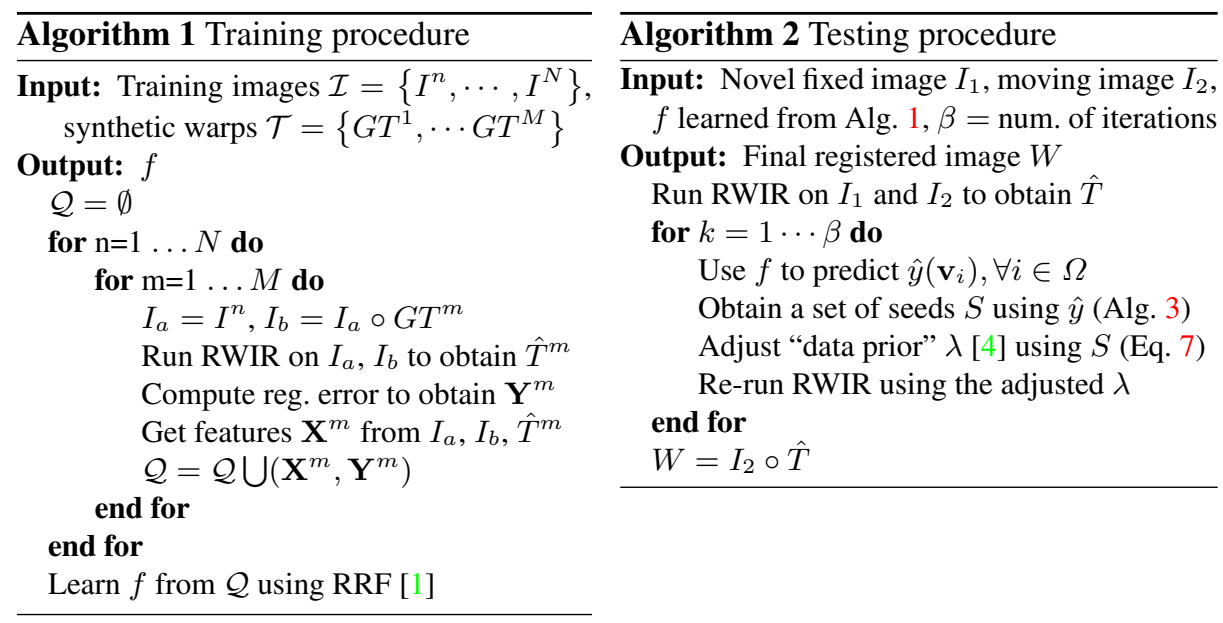

\subsection{Training stage}

Let there be a pair of images $I_{a}$ and $I_{b}$ and their corresponding registration solution $\hat{T}$ obtained by solving Eq. 1 . Our first goal is to learn a function $f$ that predicts the registration error $y_{i}$ at $\mathbf{v}_{i}$ from a set of $K$ features $\mathbf{f}_{i}$ extracted from $\hat{T}, I_{a}$, and $I_{b}$ at $\mathbf{v}_{i}$ :

$$
f\left(\mathbf{f}_{i} ; \hat{T}, I_{a}, I_{b}\right)=\hat{y}\left(\mathbf{v}_{i}\right) \equiv \hat{y}_{i} .
$$

We modeled $f$ using regression random forests (RRF) [1]. While other training methods are possible, e.g. support vector machines, we chose random forests because they 1) additionally infer the importance of features, 2) remove outliers with high accuracy, 3) resistant to over-fitting, 4) can be trained efficiently, even when thousands of input features are used, and 5) requires no feature normalization procedures [1].

Data collection. Our training data $\mathcal{Q}$ of paired input and output matrices is constructed from a set of images $\mathcal{I}$ and synthetically generated warps $\mathcal{T}$. Specifically, for each image $I_{a} \in \mathcal{I}$ and the $m$-th warp $G T^{m} \in \mathcal{T}$, we simulate a pair of misaligned images by generating $I_{b}$ as $I_{a} \circ G T^{m}$. We then deformably register $I_{a}$ and $I_{b}$ by solving Eq. 1 to obtain $\hat{T}^{m}$. Next, at each location $i$, we extract a set of image-based and deformation-based features $\mathbf{f}_{i}$ from $\hat{T}^{m}, I_{b} \circ \hat{T}^{m}$, and $I_{a}$ (details later). We then concatenate feature values from all locations to form a feature matrix $\mathbf{X}^{m}$ that corresponds to the $m$-th simulation. Finally, we calculate the pointwise registration error of $\hat{T}^{m}$, i.e. $y\left(\mathbf{v}_{i}\right)=\left\|\hat{T}^{m}\left(\mathbf{v}_{i}\right)-G T^{m}\left(\mathbf{v}_{i}\right)\right\|$, and concatenate $y$ from all locations to form the corresponding output array $\mathbf{Y}^{m}$. Repeating this procedure over the $N$ images and $M$ warps thus forms our training set.

Our feature set, $\mathcal{F}$, consists of deformation-based and image-based features $(K \leq$ 105). We explored 4 sets of image-based features: 1) the squared intensity difference between each registered pair; 2) their sum of squared intensity difference (SSD) over 
patches,

$$
S S D_{\text {patch }}\left(\mathbf{v}_{i}\right)=\sum_{\mathbf{v}_{s} \in \mathcal{N}\left(\mathbf{v}_{i}\right)} I_{a}\left(\mathbf{v}_{s}\right)-I_{b} \circ G T\left(\mathbf{v}_{s}\right),
$$

where $\mathcal{N}$ is the neighborhood of $\mathbf{v}_{i}$ of size $\left.3^{d} ; 3\right)$ their difference in intensity gradient magnitude, and 4) the difference between their MIND descriptors [6]. Deformationbased features include the determinant of Jacobian of $\hat{T}$, denoted as $J$, and our proposed uncertainty measure which we now present.

As motivated in Sec. 1, Shannon's entropy would not take into account the spatial information inherit to the displacement labels, and as we show in [10], it gives unintuitive results in the registration context. In search of alternatives, we explored the option of fitting various continuous probabilistic distributions over the obtained label space prior to standard Shannon's entropy calculation or K-nearest neighbour estimation [10]. A few other definitions were also examined, although all aforementioned options were suboptimal. To this end, we developed a novel uncertainty measure that is defined as the expectation of the registration error given the probabilistic field $\mathcal{P}$ (defined over labels) derived by solving Eq. 1 . In particular, we first assume that each $\ell_{i}$ is a potential ground truth and so, its expected error is $\mathrm{E}\left[\delta_{i}\right]=\sum_{j} p_{j} \delta_{i j}$, where $\delta_{i}$ is a random variable for the Euclidean distance to $\ell_{i}$ and $\delta_{i j}=\left\|\ell_{j}-\ell_{i}\right\|$. If the probability of $\ell_{i}$ being a ground truth is $p_{i}$, then we obtain:

$$
U_{e x p}=\sum_{i} p_{i}\left[\mathrm{E}\left[\delta_{i}\right]\right]=\sum_{i} p_{i} \sum_{j} p_{j}\left\|\ell_{i}-\ell_{j}\right\| .
$$

Now, if we consider only the maximum a posteriori (MAP) label $\ell_{M A P}$, then we obtain its expected error to the ground truth. We thus propose a measure of registration uncertainty $U$ as:

$$
U=\sum_{i=1}^{L} p_{i}\left(\left\|\ell_{i}-\ell_{M A P}\right\|\right)
$$

In [10], we show that $U$ exhibits strong correlation with target registration error, and out of all options explored in [10], we find $U$ to be the most applicable for error prediction in registration. In Sec. 3, we will compare our proposed uncertainty measure with Shannon's entropy in the context of registration and their effects on registration error prediction.

Learning $f$. For ease of training [12], we quantized $y$ into 3 classes:
1) Class 1: $y\left(\mathbf{v}_{i}\right)<0.1 y_{\max }$
(low error)
2) Class 2: $0.1 y_{\max } \leq y\left(\mathbf{v}_{i}\right) \leq 0.6 y_{\max }$ (medium error)
3) Class 3: $y\left(\mathbf{v}_{i}\right)>0.6 y_{\max }$
(high error)

where $y_{\max }$ is the maximum registration error of each training trial (and again, $y$ is the known error). We then trained $f$ using RRF of 80 trees.

\subsection{Testing stage}

We develop an iterative registration method based on prior learning that steers the registration process towards minimizing registration error as predicted by $f$. Specifically, for a new pair of images and a tentative registration solution, we extract the set of features 

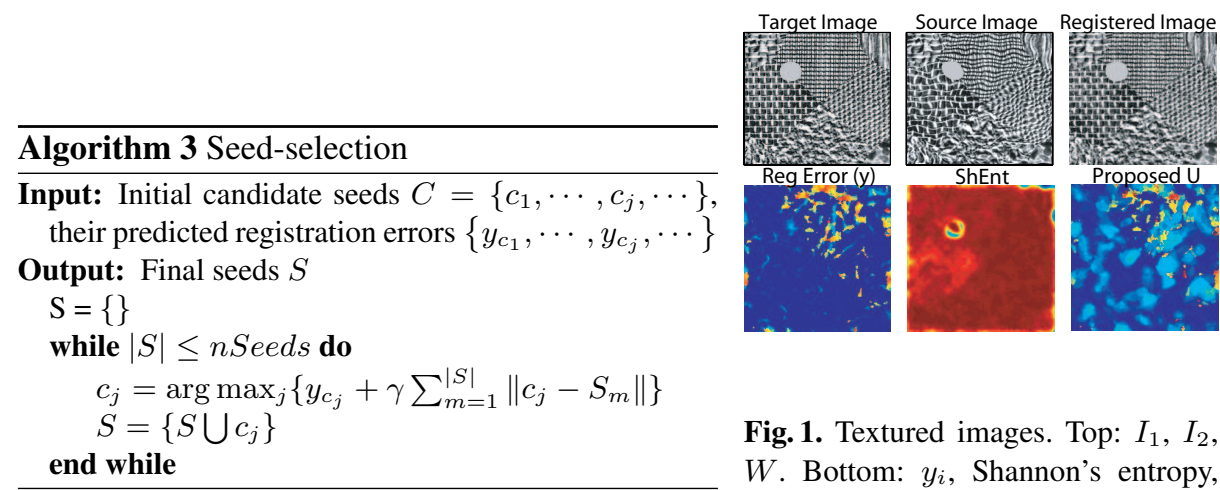

Fig. 1. Textured images. Top: $I_{1}, I_{2}$, $W$. Bottom: $y_{i}$, Shannon's entropy, and proposed $U$.

presented in Sec. 2.1 and employ $f$ to locate regions with low predicted registration error (class 1). The current solution derived at these locations are highly likely to be valid spatial correspondences and thus are regarded as trustworthy. Our reinforcementapproach reinforces these spatial correspondences and leverages them to influence the solution in the next iteration. We next explain how we locate these confident regions and how their correspondences are reinforced.

Reinforcement. Using terminology from [3,4,5], locations of low predicted error will act as candidate seeds, i.e. nodes that will be given preferred spatial correspondences. Given an initial set of locations with low predicted error (as determined by thresholding $y$ ), we short-listed them using Alg. 3 such that the final candidates are as spatially distributed as possible.

Having obtained the candidate seeds $S$, the next step is to encode them in RWIR. One way is to explicitly enforce them as boundary conditions [5]. However, this assumes that the preferred correspondences are perfect. To relax this assumption, we employ a soft approach, where the "data prior" [5], denoted as $\lambda$, is adjusted so that the preferred correspondences are encouraged, rather than strictly enforced. Thus, we compute the data prior at $\mathbf{v}_{i}$ for label $l$ as:

$$
\lambda_{i}^{l}= \begin{cases}1 & \text { if } v_{i} \in S \text { and } f \text { predicts error } \hat{y}_{i} \text { to be of Class } 1 \\ 0 & \text { if } v_{i} \in S \text { and } f \text { predicts error } \hat{y}_{i} \text { to be of Class } 3 \\ \exp \left(-D_{M I N D}\left(I\left(\mathbf{v}_{i}\right), J\left(\mathbf{v}_{i}+\ell_{i}\right)\right)\right. & \text { otherwise }\end{cases}
$$

where $S$ is the set of seeds chosen from Alg. 3 and $D_{M I N D}$ denotes the Euclidean distance between the MIND descriptors [6] extracted from $I$ and $J$.

\section{Results}

Materials. We performed synthetic and real experiments. In the synthetic case, 100 pairs of MRI brain images were used in the training stage. The learned $f$ was then evaluated on 150 image pairs. Image pairs were created by applying random warps to the fixed images. For the real experiments, 90 pairs of $3 D$ MRI brain images were registered; evaluation was done based on their registered segmentation maps. 


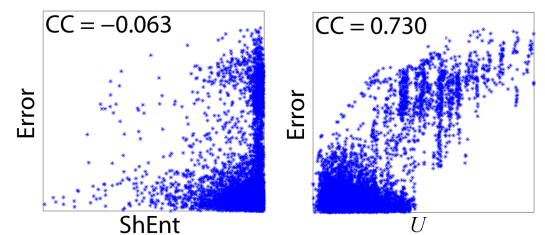

Fig. 2. Scatter plot and correlation coefficient (CC) between $y$ and $U$ as measured from trials involving syn. data.

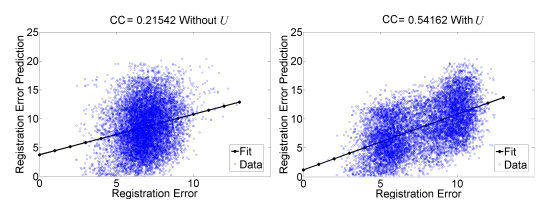

Fig. 3. Scatter plot and CC between real and predicted registration errors ( $y$ and $\hat{y}$ ) when the proposed $U$ uncertainty measure was (a) omitted from, or (b) included in $\mathcal{F}$.

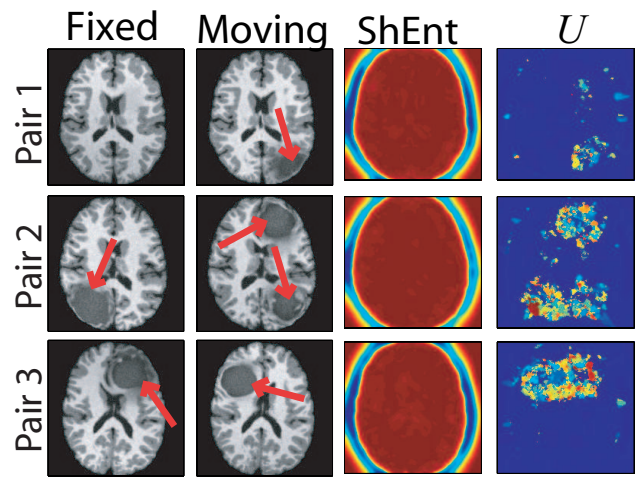

Fig. 4. Ability of $U$ in identifying misregistrations. From left to right: $I_{1}, I_{2}$, ShEnt, and $U$. Due to presences of tumors, regions with missing correspondences are highly uncertain. While ShEnt has failed to detect regions of high uncertainty in $\hat{T}$, our proposed method reflected the uncertainty in $\hat{T}$ at these regions.

Exp. 1: Usefulness of $U$. We first examine how this feature correlates with registration error $y$. For synthetic data, we created a synthetic texture image and corrupted it by adding to it a homogeneous region so that the lack of image features in this region will create ambiguity in the registration solution, thereby increasing uncertainty in this region. Then, we generated a set of moving images by applying synthetic warps to the fixed image. The warps were created by randomly perturbing the control points of a B-spline deformation grid whose displacements magnitude followed $\mathcal{N}(6,2)$ voxels. We calculated the registration error $(y)$ and $U$ using the derived label probabilities and subsequently, their overall correlations. As shown in Fig. 2, our proposed measure $U$ exhibits much higher correlation with actual error than Shannon's entropy (ShEnt). For real data, we used images from a public database ${ }^{1}$ and examined the ability of $U$ in detecting misregistrations caused by missing correspondences (due to the presences of brain tumors). Example results are shown in Fig. 4. Examining the results visually, we find that ShEnt has failed to detect misregistrations caused by the presence of tumor (nor uncertainty of the solution in these regions), while $U$ was able to reflect uncertainty (registration errors) much more effectively. Fig. 1 also shows similar result when we employed synthetically generated textured images.

Exp. 2: $U$ improves prediction accuracy of $f$. We next examined how the addition of $U$ as feature in $\mathcal{F}$ can improve accuracy of error prediction. For this experiment, we applied a synthetic warp to a target image and registered it to a source image in an attempt to recover the known warp. Fig. 3 shows a scatter plot of the actual $(y)$ and

\footnotetext{
${ }^{1}$ http://www2.imm.dtu.dk/projects/BRATS2012/
} 

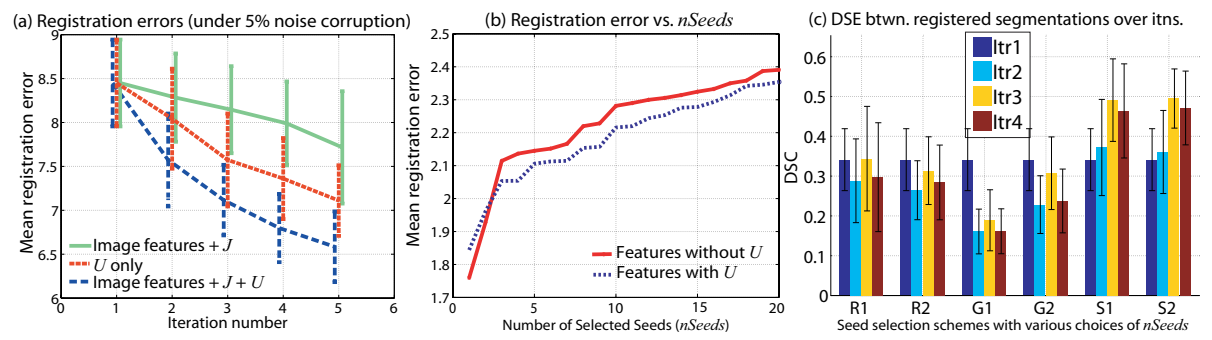

Fig. 5. (a) Registration error over iterations for different feature subsets. It can be seen that the use of image features with $J$ only (green curve) was inferior to use of $U$ alone (red). When all 105 features were used, the reduction was most significant (blue), i.e. as much as by $25 \%$. (b) Inclusion of $U$ always decreased registration error, except when the number of seeds used was inadequate (less than 3). (c) Results of segmentation-based evaluation (Exp. 4). R1 and R2 (G1 and G2) denote addition of 5 and 20 seeds per iteration, respectively.

predicted registration errors $(\hat{y})$. Note that the correlation coefficient between $\hat{y}$ and $y$ was 0.22 when $U$ was omitted from $\mathcal{F}$, but increased to 0.54 when it was included.

Exp. 3: Evaluation of proposed learning-based RWIR. We next examined how our registration algorithm, when incorporated with prior-learning improves registration over iterations. Our evaluation setup was as follows. We employed 40 MRI brain images from the LONI Probabilistic Brain Atlas (LPBA40) dataset ${ }^{2}$ where each image is accompanied with an expert's segmentation of 56 brain structures. Then, we constructed training and validation data; the GT warps were created by randomly perturbing the control points of a B-spline deformation grid whose displacement magnitudes followed $N(6 ; 2)$. This resulted in 250 pairs of synthetically misaligned images with GT registrations. The entire data set was then split into a training set of size 100 and a validation set of size 150, where the former was used to learn and test $f$, while the latter was used to quantify the registration accuracy achieved by our method as evaluated on unseen image pairs. For reference, we also repeated the trials by training $f$ with different feature subsets. Results are shown in Fig. 5a where we can see that the decrease in registration error is monotonic. Further, we see that the inclusion of $U$ in the feature set improved the error reduction rate. We then examined whether this effect was due to differences in the number of seeds used ( $n$ Seeds in Alg. 3). However, from Fig. 5b, we can see that the inclusion of $U$ had almost always led to greater reduction in error, except only when fewer than 3 seeds were used.

Exp. 4: Overall evaluation on real registrations. We next evaluated our proposed method on real registrations using the LPBA40 brain images. Since there is no known GT for these images, we calculated the Dice similarity coefficient (DSC) of the corre-

\footnotetext{
${ }^{2}$ http://www.loni.ucla.edu/
} 
sponding registered segmentations to evaluate the obtained solutions. For reference, we also compared our proposed uncertainty-based seed-selection strategy (Alg. 3) against other potential alternatives: 1) random seed selection (R), which selects seeds at random, 2) grid-based seed selection (G), which selects seeds at regular interval and mimicks how users choose landmark correspondences by scrolling through slices in the volume. Results are shown in Fig. 5c, where we plotted the DSC after registration obtained by the schemes presented above. Evidently, our proposed method gave the highest final DSC.

\section{Conclusions}

We have proposed a novel uncertainty measure for evaluating probabilistic registration solutions and incorporated this measure into a self-reinforcing registration framework that consists of 1) learning a registration error prediction function and 2) certainty-based seed-selection strategy for reinforcing trusted spatial correspondences in a tentative solution. As future work, we will extend our evaluation experiments with more data and demonstrate applicability of the proposed uncertainty measure in radiation therapy.

Acknowledgements. The authors are grateful to Brian Booth for his input and Natural Sciences and Engineering Research Council and NeuroDevNet for their partial funding.

\section{References}

1. L. Breiman. Bagging predictors. Mach. Learn., 24(2):123-140, Aug. 1996.

2. D. Cobzas and A. Sen. Random walks for deformable image registration. In MICCAI, pages 557-565, 2011.

3. C. Couprie, L. Grady, L. Najman, and H. Talbot. Power watershed: A unifying graph-based optimization framework. IEEE Trans. Pattern Anal. Mach. Intell., 33(7):1384-1399, 2011.

4. L. Grady. Multilabel random walker image segmentation using prior models. IEEE CVPR, pages 763-770, 2005.

5. L. Grady. Random walks for image segmentation. IEEE Trans. Pattern Anal. Mach. Intell., 28(11):1768-1783, Nov. 2006

6. M. Heinrich, M. Jenkinson, M. Bhushan, T. Matin, F. Gleeson, M. Brady, and J. Schnabel. MIND: Modality independent neighbourhood descriptor for multi-modal deformable registration. Medical Image Analysis, 16(7):1423-1435, 2012.

7. T. Kohlberger, V. Singh, C. Alvino, C. Bahlmann, and L. Grady. Evaluating segmentation error without ground truth. In MICCAI, pages 528-536, 2012.

8. J. Kybic. Bootstrap resampling for image registration uncertainty estimation without ground truth. IEEE Trans. Image Process., 19(1):64-73, 2010.

9. J. Kybic and C. Nieuwenhuis. Bootstrap optical flow confidence and uncertainty measure. Computer Vision and Image Understanding, 115(10):1449-1462, 2011.

10. T. Lotfi. Uncertainty in probabilistic image registration. Master's thesis, Simon Fraser University, Burnaby, Canada, 2013.

11. S. Muenzing, K. Murphy, B. Ginneken, and J. Pluim. Automatic detection of registration errors for quality assessment in medical image registration. SPIE Medical Imaging: Image Processing, 7259:1-9.

12. S. Muenzing, B. van Ginneken, and J. P. W. Pluim. DIRBoost: An algorithm for boosting deformable image registration. In Biomedical Imaging (ISBI), pages 1339-1342, 2012.

13. T. Tang and A. Chung. Non-rigid image registration using graph-cuts. In MICCAI, pages 916-924. 2007. 